NOTA

\title{
REPORTE DE LA MEDUSA Aglauropsis kawari (CNIDARIA, HYDROZOA, LIMNOMEDUSAE) PARASITADA CON METACERCARIA DE Monascus filiformis (DIGENEA, FELLODISTOMIDAE) EN EL ESTUARIO DEL RÍO DE LA PLATA, URUGUAY
}

\author{
Marcia Lauria Cruz* \& Gabriela Failla Siquier \\ Laboratorio de Zoología de Invertebrados, Departamento de Biología Animal, Facultad de Ciencias, Iguá 4225 \\ Montevideo 11400, Uruguay.
}

Autor para correspondencia: rlauria @fcien.edu.uy

\section{RESUMEN}

Se reporta el hallazgo en el estuario del Río de la Plata de Aglauropsis kawari ampliándose su distribución geográfica a la costa del Departamento de Maldonado, Uruguay. Asimismo, se halló una medusa parasitada por una metacercaria de Monascus filiformis lo cual constituye un nuevo registro de hospedador para dicho trematodo.

Palabras clave: Hydrozoa, Aglauropsis kawari, Monascus filiformis, Río de la Plata.

\section{ABSTRACT \\ Report of the Aglauropsis kawari jellyfish (Cnidaria, Hydrozoa, Limnomedusae) parasitated with the metacercaria of Monascus filiformis (Digenea, Fellodistomidae) in the estuary of the Rio de la Plata, Uruguay. The finding of Aglauropsis kawari in the estuary of the Río de la Plata is reported, expanding its geographical distribution to the coast of Maldonado Department, Uruguay. Also, a jellyfish parasitized by a metacercaria of Monascus filiformis was found, which constitutes a new host for this trematode.}

Keywords: Hydrozoa, Aglauropsis kawari, Monascus filiformis, Río de la Plata.

La gran mayoría de las publicaciones de los organismos gelatinosos (Medusozoa) para el Uruguay se enmarcan dentro de estudios realizados para el Atlántico Sudoccidental Bouillon, 1999; Failla Siquier, 2006; Genzano et al., 2008; Leoni et al., 2016; Schiariti et al., 2018) y en el caso de las medusas de Hydrozoa los estudios son escasos o bien menciones aisladas, en particular en los últimos años éstos están enfocados principalmente a su rol como hospedadores intermediarios de parásitos digeneos (Failla Siquier \& Díaz Briz, 2013; Failla Siquier, 2014; Failla Siquier et al., 2018). Dentro de la familia Olindiidae, el género Aglauropsis comprende las siguientes especies: $A$. conanti Browne, 1902 hallada en las Islas Malvinas y áreas adyacentes; $A$. edwarsii Pagés, Bouillon \& Gili,
1991 citada para la costa de África suroccidental; $A$. kawari Moreira \& Yamashita, 1972 para el Atlántico Sur. A. agassazi Müller, 1865 fue encontrada en las costas de Brasil, pero al no contar con una descripción adecuada fue considerada como $A$. kawari Moreira \& Yamashita, 1972. En el presente trabajo se cita a $A$. kawari en la costa estuarina de Maldonado, Uruguay, con uno de los ejemplares parasitado por una larva (metacercaria) del trematodo digeneo Monascus filiformis.

Nueve ejemplares de $A$. kawari fueron colectados en la costa del balneario Las Flores, Departamento de Maldonado, Uruguay (34050'S - 55'25'O) con red de mano de $500 \mu$ en diciembre de 2016 , octubre y diciembre de 2017, diciembre de 2018 y en enero de 2019. Se encontraron junto con las hidromedusas Eucheilota maculata, Clytia hemisphaerica y Liriope tetraphylla. El rango de temperatura y salinidad fue entre $17^{\circ} \mathrm{C}-22^{\circ} \mathrm{C}$ y $25-29$ respectivamente (Tabla 1 ). Las muestras se fijaron en formaldehído al $5 \%$ en agua de mar.

Las características que describen $A$. kawari según Moreira y Yamashita (1972) son: umbrela más ancha que alta (3.0-5.5 mm de ancho y 2.0-4.0 mm de altura), con forma de cuenco en ejemplares fijados. Velo ancho. Estómago cuadrangular, aproximadamente la mitad

Tabla 1. Fecha de colecta de A. kawari, registro de temperatura (ํ) y salinidad del agua en el balneario Las Flores, Dpto. de Maldonado, Uruguay. $\left(^{*}\right)$ : hallazgo de la medusa parasitada por M. filiformis; SR: sin registro.

\begin{tabular}{ccc} 
Fecha & Temperatura del agua & Salinidad \\
\hline $8 / 10 / 2016^{*}$ & 18 & SR \\
$3 / 12 / 2016$ & 19 & 25 \\
$2 / 12 / 2017$ & 17 & 29 \\
$27 / 12 / 2018$ & 22 & 25 \\
\hline
\end{tabular}




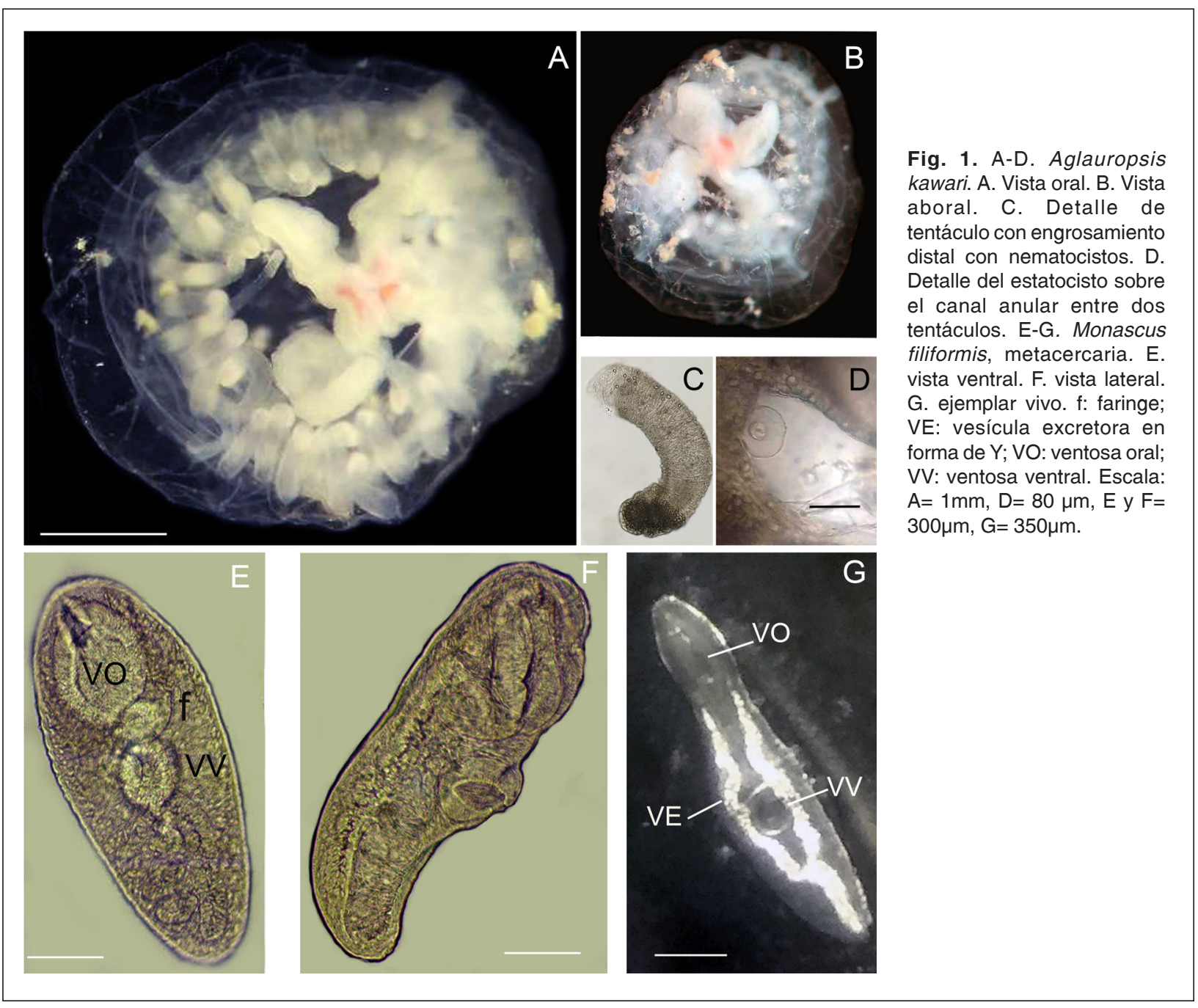

de la longitud de la cavidad subumbrelar; boca con 4 labios simples perradiales; canales radiales anchos; gónadas en forma de bolsa, que cuelgan de la parte proximal de los canales radiales y se continúan con las esquinas de la pared del estómago; gónadas y estómago de color rojizo en los ejemplares vivos, esta coloración puede desvanecerse con el tiempo en ejemplares fijados (Fig. 1A y B). Individuos adultos con hasta 40 tentáculos dispuestos en dos hileras, 20 por encima del margen de la campana (de mayor longitud) y 20 directamente en el margen de la campana (de menor longitud), todos presentan engrosamiento distal con nematocistos (Fig. 1C); 20 estatocistos (Fig. 1D). Los ejemplares hallados tienen un promedio de 3 $\mathrm{mm}$ de diámetro y $2 \mathrm{~mm}$ de altura de la campana, con 28 a 39 tentáculos. Pólipo desconocido.

Una metacercaria fue encontrada en uno de los ejemplares (colectada en diciembre de 2016) embebida en la campana con las siguientes características: cuerpo oval, subcilíndrico, entre 370 a $450 \mu \mathrm{m}$ de largo y 229 a $239 \mu \mathrm{m}$ de ancho (en material fijado); ventosa oral subterminal, ventosa ventral (acetábulo) en la zona ecuatorial; faringe muscular, intestino con un ciego pequeño izquierdo y uno derecho que llega hasta el extremo posterior del cuerpo; primordios del aparato reproductor constituidos por un par de testículos, ovario detrás de la ventosa ventral (acetábulo pretesticular); vesícula excretora muy notoria en forma de "Y". De acuerdo a Girola et al. (1992), correspondería a la especie Monascus filiformis (Fig. 1E-G). Esta larva también ha sido encontrada parasitando a las hidromedusas $L$. tetraphylla, $C$. hemisphaerica, E. maculata y Bougainvillia pagesi colectadas en la misma localidad (Failla Siquier \& Díaz Briz, 2013; Failla Siquier, 2014; Failla Siquier et al., 2018) y en la región del Océano Atlántico Sudoccidental (Girola et al., 1992; Díaz Briz et al., 2012).

Como ocurre en la mayoría de los animales planctónicos, el endemismo en medusas es raro y las 
especies hasta ahora halladas en la costa uruguaya presentan una amplia distribución geográfica (Failla Siquier, 2006). Sin embargo, A. kawari es endémica del Atlántico Sudoccidental (Bouillon, 1999; Migotto et al., 2002), encontrándose desde la costa de Rio Grande do Sul hasta el sur de la Provincia de Buenos Aires (Rodríguez, 2012) incluyendo el estuario del Río de la Plata (presente estudio).

\section{REFERENCIAS}

Bouillon J.F. 1999. Hydromedusae. In: D. Boltovskoy (Ed.) South Atlantic Zooplankton. Backhuys Publishers, Leiden, The Netherlands, pp.: 385465.

Díaz Briz L.M., Martorelli S.R., Genzano G.N. \& Mianzan H.W. 2012. Parasitism (Trematoda, Digenea) in medusae from the southwestern Atlantic Ocean: medusa hosts, parasite prevalences, and ecological implications. Hydrobiologia, 690: 215- 226.

Failla Siquier M.G. 2006. Zooplancton gelatinoso de la costa uruguaya. Menafra, Rodríguez - Gallego, Scarabino \& Conde (Eds.). En: Bases para la Conservación y el Manejo de la Costa Uruguaya. pp.: 97-103, Vida Silvestre, Montevideo, Uruguay.

Failla Siquier M.G. 2014. Hallazgo de la medusa Bougainvillia pagesi (Cnidaria, Hydrozoa, Anthoathecata) parasitada con metacercarias de Monascus filiformis (Digenea, Fellodistomidae) en el estuario del río de La Plata, Uruguay. Boletín de la Sociedad Zoológica del Uruguay, 23: 43-47.

Failla Siquier M.G. \& Díaz Briz L. 2013. Ocurrencia de parásitos digeneos en hidromedusas (Cnidaria, Hydrozoa) de la costa de Uruguay. XV Congreso Latinoamericano de Ciencias del Mar (COLACMAR): Primer Simposio Latinoamericano de Cnidaria: pg. 91. Punta del Este, Uruguay.

Failla Siquier M.G., Díaz Briz L. \& Lauria Cruz R. 2018. Monascus filiformis y Opechona sp.
(Digenea: Trematoda) parasitando a Eucheilota maculata y Clytia hemisphaerica (Cnidaria: Hydrozoa) en la costa estuarina de Uruguay. V Congreso de Zoología (VCUZ): pg. 84. Facultad de Ciencias. Montevideo, Uruguay.

Genzano G., Mianzan H. \& Bouillion J. 2008. Hydromedusae (Cnidaria: Hydrozoa) from the temperate southwestern Atlantic Ocean: a review. Zootaxa, 1750: 1-18.

Girola C.V., Martorelli S.R. \& Sardella N.H. 1992. Presencia de metacercarias de Monascus filiformis (Digenea, Fellodistomidae) en hidromedusas del Océano Atlántico Sur. Revista Chilena de Historia Natural, 65 (4): 409-415.

Leoni V., González S., Ortega L., Scarabino F., Failla Siquier G., Dutra A., Rubio L., Abreu M., Serra, W., Alonzo A., Stampar S.N. \& Morandini A.C. 2016. Tamoya haplonema (Cnidaria: Cubozoa) from Uruguayan and adjacent waters: oceanographic context of new and historical findings. Marine Biodiversity Records, 9 (1): $92-$ 97.

Migotto A.E., Marques A.C., Morandini A.C. \& Silveira F.L.D. 2002. Checklist of the Cnidaria Medusozoa of Brazil. Biota Neotropica, 2 (1): 1-31.

Moreira G.S. \& C. Yamashita. 1972. Aglauropsis kawari (Limnomedusae: Olindiasidae), a new species from the South Atlantic Ocean. Marine Biology, 14 (3): 271-274.

Rodriguez C.S. 2012. Hidromedusas del Atlántico Sudoccidental: biodiversidad y patrones de distribución (Doctoral dissertation, PhD thesis. Mar del Plata: Universidad Nacional de Mar del Plata, Argentina), $199 \mathrm{pp}$.

Schiariti A., Dutto M.S., Pereyra D.Y., Failla Siquier G. \& Morandini C.A. 2018. Medusae (Scyphozoa and Cubozoa) from southwestern Atlantic and Subantarctic region $\left(32-60^{\circ} \mathrm{S}, 34-70^{\circ} \mathrm{W}\right)$ : species composition, spatial distribution and life history traits. Latin American Journal of Aquatic Research, 46 (2): 240-257.

Fecha de Recepción: 14 de mayo de 2019 Fecha de Aceptación: 11 de setiembre de 2019 